
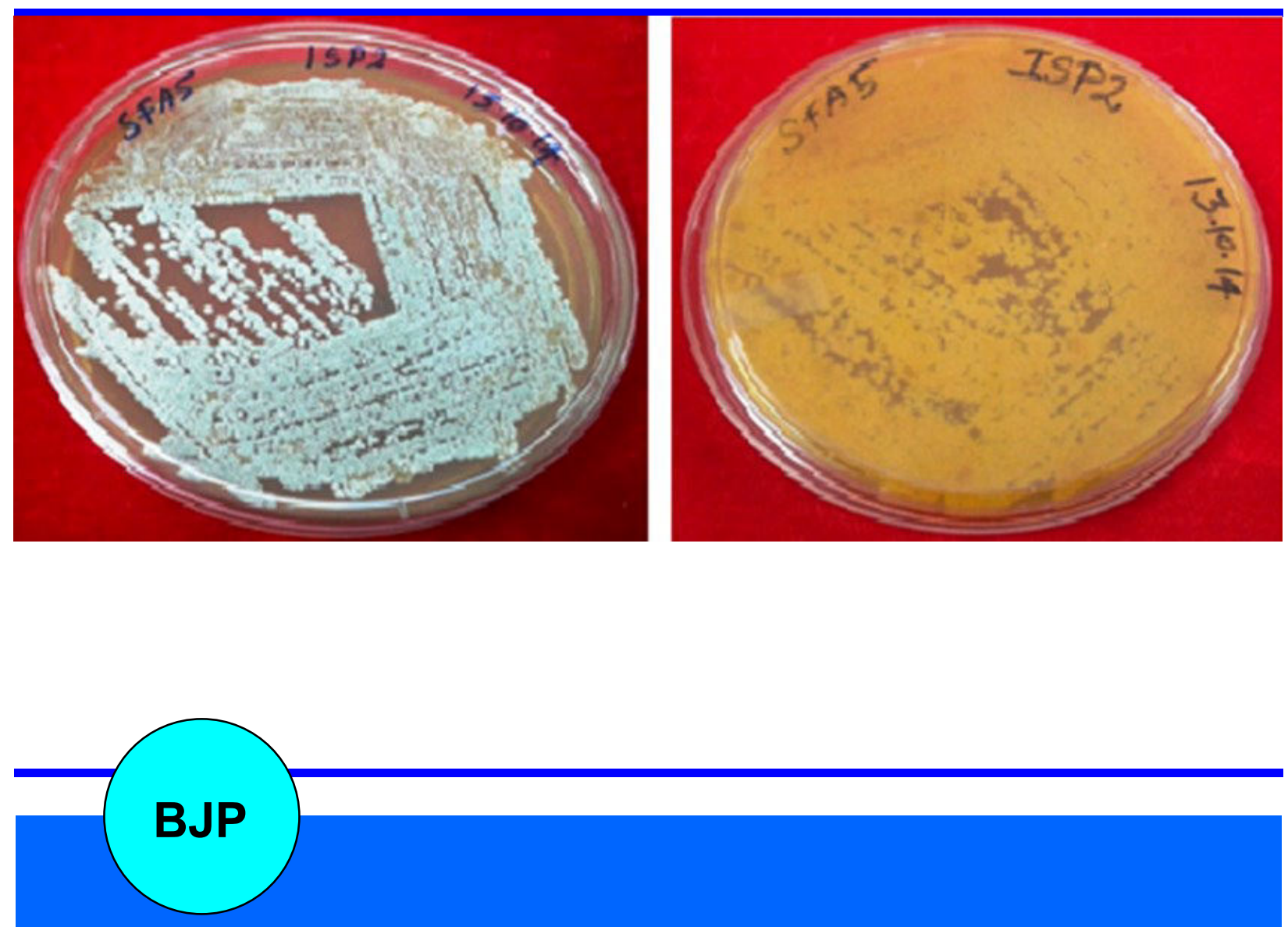

Bangladesh Journal of Pharmacology

Research Article

HPTLC fingerprint profile of antibacterial compound produced from forest soil Streptomyces SFA5 
Abstracted/indexed in Academic Search Complete, Asia Journals Online, Bangladesh Journals Online, Biological Abstracts, BIOSIS Previews, CAB Abstracts, Current Abstracts, Directory of Open Access Journals, EMBASE/Excerpta Medica, Google Scholar, HINARI (WHO), International Pharmaceutical Abstracts, Open J-gate, Science Citation Information Expanded (SCIE), SCOPUS and Social Sciences Citation Index; ISSN: 1991-0088

\section{HPTLC fingerprint profile of antibacterial compound produced from forest soil Streptomyces SFA5}

\section{Ponnuswamy Shekar'1, Kannaiyan Sathish Kumar'1, Venugopal Gopikrishnan² and Manikkam Radhakrishnan²}

${ }^{1}$ Department of Chemical Engineering, SSN College of Engineering, Chennai 603110 , India; ${ }^{2}$ Centre for Drug Discovery and Development, Sathyabama University, Chennai 600 119, India.

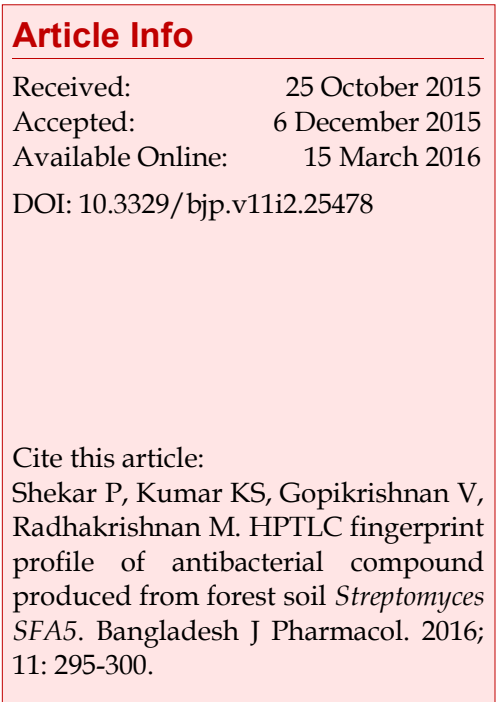

\begin{abstract}
The present study investigates the presence of antimicrobial compounds from potent isolate Streptomyces sp. SFA5 from the Sabarimala forest ecosystem, Western Ghats, Kerala, India. Preliminary screening revealed Streptomyces sp. SFA5 isolate shows significant inhibition against the bacterial and fungal pathogens. The antibacterial compound from isolate SFA5 was produced by submerged fermentation using yeast extract malt extract broth and extracted using different solvents. Among the different solvent extracts tested, the ethyl acetate extract of SFA5 shows maximum zone of inhibition against Staphylococcus aureus $(23 \mathrm{~mm})$ and Bacillus cereus $(24 \mathrm{~mm})$. A simple, sensitive and accurate HPTLC method has been performed for the quantitative estimation of bioactive compounds from ethyl acetate extract of Streptomyces sp. SFA5. The optimized solvent system for mobile phase in HPTLC was toluene: ethyl acetate: methanol: acetic acid (5:3:1:0.5, v/v/v/v). Results of HPTLC finger printing of ethyl acetate extract of the strain SFA5 at UV 254 and 366 with corresponding $\mathrm{R}_{\mathrm{f}}$ values substantiate the presence of bioactive compounds.
\end{abstract}

\section{Introduction}

Microbial natural products continue to represent as important route for the discovery of novel chemicals for the development of new therapeutic agents (Skoko et al., 2005). Among the microbial producers, actionbacteria holds a prominent position as targets in screening program due to their diversity and account for the production of most of discovered bioactive secondary metabolites, primarily antibiotics, immunosuppressive agents, enzymes and enzyme inhibitors (Thakur et al., 2007). Due to uniqueness, large geographic variation, different soil types and their contents of forest, it is quite like that there is a vast distribution of antibiotic producing Actinobacteria in forest environment (Aravamuthan et al., 2010).

High performance thin layer chromatography (HPTLC) is one of the modern sophisticated techniques that can be used for quick and easy determination of quality, authenticity and purity of the natural extracts, crude drugs and market formulations (Mamatha et al., 2011; Miyadoh et al., 1993). HPTLC provides valuable tool for reliable identification through chromatographic fingerprints that can be visualized and stored as electronic images.

The present study reports the HPTLC fingerprint profile and qualitative analysis of bioactive compounds produced by soil Streptomyces sp. SFA5 isolated from the Sabarimala forest ecosystem, Western Ghats, India.

\section{Materials and Methods}

\section{Description of Streptomyces species SFA5}

Streptomyces species (SFA5) was isolated from Sabari- 
mala Forest Ecosystem, Western Ghats, Kerala, India (Latitude: $09^{\circ} 25^{\prime} 59^{\prime \prime} \mathrm{N}$; Longitude: $77^{\circ} 04^{\prime} 59^{\prime \prime} \mathrm{E}$ ) using starch casein agar medium supplemented with filter sterilized nystatin $(20 \mu \mathrm{g} / \mathrm{mL})$ and nalidixic acid (100 $\mu \mathrm{g} / \mathrm{mL}$ ) by serial dilution plating method. Viability of strain SFA5 was maintained on ISP2 agar slants at $4^{\circ} \mathrm{C}$ (Shekar et al., 2011). Strain SFA5 produced soluble yellow pigment on ISP2 agar medium.

\section{Production and extraction of bioactive compounds}

For the preparation of inoculum, spores of Streptomyces sp. SFA5 were inoculated into $100 \mathrm{~mL}$ yeast extract malt extract (YEME) broth in $500 \mathrm{~mL}$ conical flask at $28^{\circ} \mathrm{C}$ in rotary shaker with $90 \mathrm{rpm}$ for 7 days (Saravanan et al., 2012). After incubation the fermented broth which contains the bioactive compounds was centrifuged at $10,000 \mathrm{rpm}$ for $30 \mathrm{~min}$ at $4^{\circ} \mathrm{C}$. The cell free supernatant was tested for antimicrobial activity by agar well diffusion method (Chaudhary et al., 2013). To extract the antimicrobial compounds, cell free supernatant was extracted by liquid-liquid extraction method (Shekar et al., 2012) using equal volume of $(1: 1 \mathrm{v} / \mathrm{v})$ different solvents such as ethyl acetate, methanol, chloroform, dichloromethane, and $n$-hexane and allowed to dry. The concentrated crude extract was impregnated in sterile $5 \mathrm{~mm}$ filter disc at $100 \mu \mathrm{g}$ concentration placed over the test organism. Test organisms used for screening include standard pathogens such as Staphylococcus aureus NCIM 2079, Bacillus cereus NCIM 2106, Escherichia coli NCIM 2256, Pseudomonas aeruginosa NCIM 5031, Candida albicans ATCC 90028, Aspergillus fumigatus NCIM 6645 and Cryptococcus neoformans ATCC 66031 and clinical pathogens such as S. aureus, enteropathogenic E. coli, P. aeruginasa, K. pneumoniae and C. albicans. Zone of inhibition was measured after 24 hours of incubation at $37^{\circ} \mathrm{C}$ (Video clip).

\section{HPTLC profiling of ethyl acetate extract}

The crude ethyl acetate extract of Streptomyces sp. SFA5 which showed good activity against test pathogens was subjected to HPTLC analysis. The ethyl acetate extract was dissolved in $200 \mu \mathrm{L}$ of ethyl acetate from which one micro liter of crude extract was loaded as $6 \mathrm{~mm}$ band length in the $3 \times 10 \mathrm{~cm}$ silica gel 60F254 coated TLC plate using hamilton syringe and camag linomat 5 instrument.

\section{Spot development}

The crude extract loaded plate was kept in TLC twin trough developing chamber (after saturated with solvent vapour) with respective mobile phase and the plate was developed in the mobile phase up to $90 \mathrm{~mm}$ and it was then dried by hot air to evaporate solvents from the plate. Using UV light torch, the developed spots were marked, the distance travelled by each spot in baseline and relative $R_{f}$ values were calculated (Baskar et al., 2010).

$$
\mathrm{R}_{\mathrm{f}} \text { value }=\frac{\text { Distance travelled by the substance }}{-} \text { Distance travelled by the solvent }
$$

\section{Photo documentation}

The TLC plate was kept in photo documentation chamber and the images were taken at white light, UV $254 \mathrm{~nm}$ and UV $366 \mathrm{~nm}$. The developed TLC plates were sprayed with anisaldehyde sulfuric acid reagent and dried at $100^{\circ} \mathrm{C}$ in hot air oven. After derivatization, the TLC plate was fixed in scanner stage (CAMAG TLC SCANNER 3) and scanning was done at $366 \mathrm{~nm}$. The peak table and peak densitogram were also noted.

\section{Results}

Streptomyces sp SFA5 was showed good growth with ash white color aerial mycelium, brown color substrate mycelium and diffusible yellow pigment on YEME agar

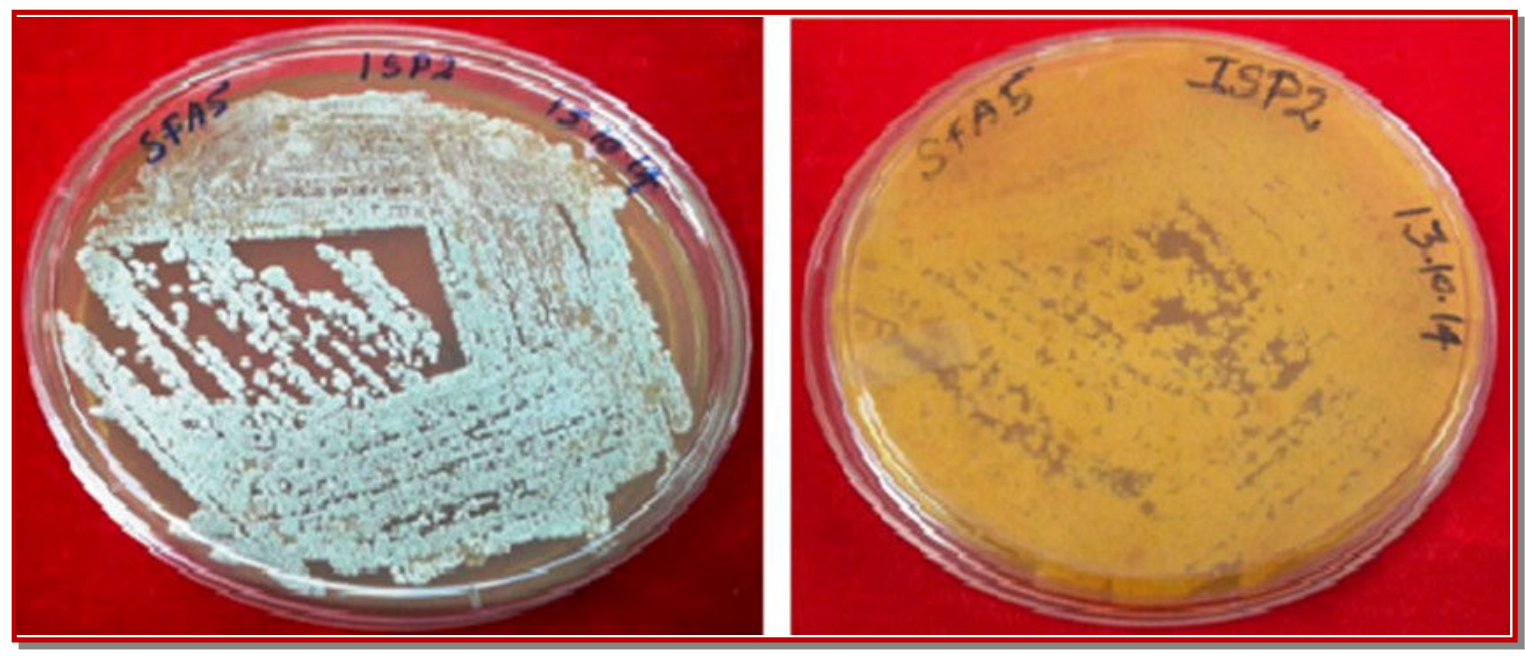

Figure 1: Morphological pattern of Streptomyces sp SFA5 
medium (Figure 1). The cell free supernatant of Streptomyces sp.SFA5 exhibited good activity over the standard as well as clinical pathogens in YEME broth (Radhakrishnan et al., 2013).

In total, the supernatant showed good activity over Gram positive bacteria and fungi than Gram negative bacteria. The supernatant of Streptomyces sp. SFA5 inhibited standard bacterial pathogen such as Staphylococcus aureus NCIM 2079 (22 mm) and Bacillus cereus NCIM $2106(23 \mathrm{~mm})$ as well clinical pathogen such as Staphylococcus aureus (19 $\mathrm{mm}$ ) with a maximum zone of inhibition (Table I). Further cell free supernatant of Streptomyces $s p$. SFA5 inhibited the standard fungal pathogen Candida albicans ATCC 90028 (18 mm) and clinical fungal pathogen Candida albicans $(22 \mathrm{~mm})$ with maximum zone of inhibition. Five different solvent tested for extraction, ethyl acetate extract of Streptomyces sp. SFA5 grown on YEME broth showed maximum zone of inhibition on antimicrobial activity, while other solvent extract such as dichloromethane, chloroform and n-hexane extract showed no zone of inhibition (Table II).

The HPTLC profiling of ethyl acetate extract of Streptomyces sp. SFA5 shows presence of antimicrobial compounds in the chromatograph as well as in UV after derivatization (Febina et al., 2013). The analysis was carried at volume of $10 \mu \mathrm{L}$ Streptomyces sp. SFA5 extract at UV $254 \mathrm{~nm}$ and UV $366 \mathrm{~nm}$ (Figure 2). Yellow brown color spots were observed after derivatization confirmed the presence of antimicrobial compound and optimized solvent system for mobile phase involves toluene: ethyl acetate: methanol: acetic acid (5:3:1:0.5, $\mathrm{v} / \mathrm{v} / \mathrm{v} / \mathrm{v})$.

The Streptomyces sp. SFA5 shows nearly 14 peaks with good Rf and area \% values for the peak 7 (0.4 and 29\%)

\section{Table I}

\section{Antimicrobial activity of Streptomyces sp. SFA5 by agar well diffusion method}

\begin{tabular}{|c|c|c|c|c|c|}
\hline \multirow{2}{*}{\multicolumn{2}{|c|}{ Test organism }} & & \multicolumn{3}{|c|}{$\begin{array}{l}\text { Zone of inhibition } \\
\text { (mm in diameter) }\end{array}$} \\
\hline & & & \multicolumn{3}{|c|}{ YEME broth } \\
\hline \multicolumn{6}{|l|}{ Standard pathogen } \\
\hline \multicolumn{2}{|c|}{ Staphylococcus aureus NCIM 2079} & & \multicolumn{3}{|c|}{22} \\
\hline \multicolumn{2}{|c|}{ Bacillus cereus NCIM 2106} & & \multicolumn{3}{|c|}{23} \\
\hline \multicolumn{2}{|c|}{ Escherichia coli NCIM 2256} & & \multicolumn{3}{|c|}{-} \\
\hline \multicolumn{2}{|c|}{ Pseudomonas aeruginosa NCIM 5031} & & \multicolumn{3}{|c|}{ - } \\
\hline \multicolumn{2}{|c|}{ Candida albicans ATCC 90028, } & & \multicolumn{3}{|c|}{18} \\
\hline \multicolumn{2}{|c|}{ Aspergillus fumigatus NCIM 6645} & & \multicolumn{3}{|c|}{-} \\
\hline \multicolumn{2}{|c|}{ Cryptococcus neoformans ATCC 66031} & & \multicolumn{2}{|c|}{-} & \\
\hline \multicolumn{6}{|c|}{ Clinical pathogens } \\
\hline \multicolumn{2}{|l|}{ S. aureus } & & \multicolumn{2}{|c|}{19} & \\
\hline \multicolumn{2}{|c|}{ Enteropathogenic E. Coli } & & \multicolumn{2}{|c|}{-} & \\
\hline \multicolumn{2}{|l|}{ P. aeruginasa } & & \multicolumn{2}{|c|}{ - } & \\
\hline \multicolumn{2}{|l|}{ K. pneumonia } & & \multicolumn{2}{|c|}{-} & \\
\hline \multicolumn{2}{|l|}{ C. albicans } & & \multicolumn{2}{|c|}{22} & \\
\hline \multicolumn{6}{|c|}{ Table II } \\
\hline \multicolumn{6}{|c|}{ Effect of different solvents on the extraction of bioactive compounds from Streptomyces sp SFA5 } \\
\hline \multirow{2}{*}{ Test organism } & \multicolumn{5}{|c|}{ Solvent extracts } \\
\hline & $\begin{array}{l}\text { Ethyl acetate } \\
\text { extract }\end{array}$ & Methanol extract & $\begin{array}{l}\text { Dichloromethane } \\
\text { extract }\end{array}$ & $\begin{array}{l}\text { Chloroform } \\
\text { extract }\end{array}$ & n-Hexane extract \\
\hline S. aureus NCIM 2079 & 23 & 19 & - & - & - \\
\hline B. cereus NCIM 2106 & 24 & 20 & - & - & - \\
\hline E. coli NCIM 2256 & - & - & - & - & - \\
\hline P. aeruginosa NCIM 5031 & - & - & - & - & - \\
\hline
\end{tabular}




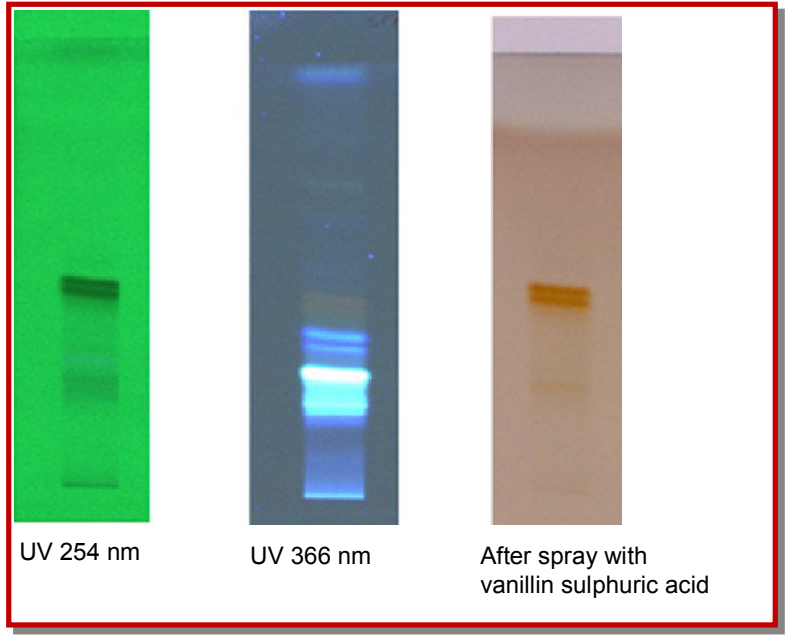

Figure 2: HPTLC profiling showing the presence of antimicrobial compound at UV 254 and UV 366 after derivatization and peak 8 (0.5 and 18\%) for finger print profile ethyl acetate extract indicates firmly the presence of antimicrobial compound at UV $254 \mathrm{~nm}$ and UV $366 \mathrm{~nm}$ (Figure 3). The Streptomyces sp. SFA5 shows nearly 6 peaks with good $\mathrm{Rf}$ and area percent values for the peak $2(0.2$ and $28 \%)$ and peak $3(0.3$ and $51 \%$ ) for finger print profile of ethyl acetate extract indicates stoutly the presence of antimicrobial compound at UV $366 \mathrm{~nm}$.

Figure 4 shows the profiling results of SFA5 ethyl acetate extract at UV $254 \mathrm{~nm}$.

\section{Discusion}

Streptomyces species SFA5 from forest ecosystem exhibited good activity against Gram positive bacteria and fungi in agar well diffusion. Most of the metabolites reported from Actinomycetes are found to be active

Track 1, DD: SFA5 Ethyl acelate extract

\begin{tabular}{|c|c|c|c|c|c|c|c|c|c|}
\hline Peak & $\begin{array}{c}\text { Start } \\
\text { Position }\end{array}$ & $\begin{array}{c}\text { Start } \\
\text { Height }\end{array}$ & $\begin{array}{c}\text { Max } \\
\text { Position }\end{array}$ & $\begin{array}{c}\text { Max } \\
\text { Height }\end{array}$ & $\begin{array}{l}\text { Max } \\
\%\end{array}$ & $\begin{array}{l}\text { End } \\
\text { End } \\
\text { Position Height }\end{array}$ & Area & $\begin{array}{c}\text { Area } \\
\%\end{array}$ & Assigned substance \\
\hline 1 & $0.01 \mathrm{Rf}$ & $0.0 \mathrm{AU}$ & $0.02 \mathrm{Rt}$ & $211.8 \mathrm{AU}$ & $11.00 \%$ & $0.06 \mathrm{Rt} 17.6 \mathrm{AU}$ & $3044.6 \mathrm{AU}$ & $6.43 \%$ & unknown * \\
\hline 2 & $0.17 \mathrm{Rf}$ & $6.1 \mathrm{AU}$ & $0.23 \mathrm{Rf}$ & 140.3AU & $7.29 \%$ & $0.24 \mathrm{Rf} 36.1 \mathrm{AU}$ & $3823.6 \mathrm{AU}$ & $8.08 \%$ & unknown ${ }^{*}$ \\
\hline 3 & $0.24 \mathrm{Rf}$ & 137.2AU & $0.25 \mathrm{Rf}$ & $154.5 \mathrm{AU}$ & $8.03 \%$ & $0.28 \mathrm{Rf} 88.9 \mathrm{AU}$ & 4046.1AU & $8.55 \%$ & unknown ${ }^{*}$ \\
\hline 4 & $0.28 \mathrm{Rf}$ & $89.4 \mathrm{AU}$ & $0.30 \mathrm{Rf}$ & $111.7 \mathrm{AU}$ & $5.81 \%$ & $0.31 \mathrm{Rf} 69.3 \mathrm{AU}$ & 2668.3AU & $5.64 \%$ & unknown ${ }^{*}$ \\
\hline 5 & $0.31 \mathrm{Rf}$ & $69.5 \mathrm{AU}$ & $0.32 \mathrm{Ri}$ & $78.4 \mathrm{AU}$ & $4.07 \%$ & $0.34 \mathrm{Rf} 48.7 \mathrm{AU}$ & $1656.7 \mathrm{AU}$ & $3.50 \%$ & unknown * \\
\hline 6 & $0.35 \mathrm{Rf}$ & $48.8 \mathrm{AU}$ & $0.37 \mathrm{Rf}$ & $64.0 \mathrm{AU}$ & $3.33 \%$ & $0.38 \mathrm{Rf} 62.7 \mathrm{AU}$ & $1537.8 \mathrm{AU}$ & $3.25 \%$ & unknown * \\
\hline 7 & $0.38 \mathrm{Rf}$ & 63.1AU & $0.45 \mathrm{Rf}$ & $448.8 \mathrm{AU}$ & $23.32 \%$ & $0.46 \mathrm{Rf} 20.2 \mathrm{AU}$ & $13717.8 \mathrm{AU}$ & $28.98 \%$ & unknown ${ }^{*}$ \\
\hline 8 & $0.46 \mathrm{Ri}$ & $422.9 \mathrm{AU}$ & $0.47 \mathrm{Rf}$ & $465.5 \mathrm{AU}$ & $24.19 \%$ & $0.50 \mathrm{Rf} 20.4 \mathrm{AU}$ & $8371.6 \mathrm{AU}$ & $17.69 \%$ & unknown ${ }^{*}$ \\
\hline 9 & $0.50 \mathrm{Rf}$ & $20.6 \mathrm{AU}$ & $0.51 \mathrm{Rt}$ & $39.8 \mathrm{AU}$ & $2.07 \%$ & $0.56 \mathrm{Rf} 17.1 \mathrm{AU}$ & $1367.4 \mathrm{AU}$ & $2.89 \%$ & unknown * \\
\hline 10 & $0.58 \mathrm{Rf}$ & $14.3 \mathrm{AU}$ & $0.61 \mathrm{Rf}$ & $26.9 \mathrm{AU}$ & $1.40 \%$ & $0.63 \mathrm{Rf} 17.0 \mathrm{AU}$ & $924.8 \mathrm{AU}$ & $1.95 \%$ & unknown * \\
\hline 11 & $0.64 \mathrm{Rt}$ & $22.8 \mathrm{AU}$ & $0.66 \mathrm{Rf}$ & $27.3 \mathrm{AU}$ & $1.42 \%$ & $0.68 \mathrm{Rf} 24.7 \mathrm{AU}$ & $719.2 \mathrm{AU}$ & $1.52 \%$ & unknown ${ }^{*}$ \\
\hline 12 & $0.69 \mathrm{Rf}$ & $25.6 \mathrm{AU}$ & $0.72 \mathrm{Rt}$ & $41.5 \mathrm{AU}$ & $2.16 \%$ & $0.75 \mathrm{Rf} 31.3 \mathrm{AU}$ & $1587.8 \mathrm{AU}$ & $3.35 \%$ & unknown * \\
\hline 13 & $0.76 \mathrm{Rt}$ & $31.0 \mathrm{AU}$ & $0.79 \mathrm{Rf}$ & $35.8 \mathrm{AU}$ & $1.86 \%$ & $0.84 \mathrm{Rf} \quad 0.8 \mathrm{AU}$ & $1436.7 \mathrm{AU}$ & $3.04 \%$ & unknown * \\
\hline 14 & $0.93 \mathrm{Rf}$ & $0.1 \mathrm{AU}$ & $0.98 \mathrm{Rf}$ & $78.2 \mathrm{AU}$ & $4.06 \%$ & $1.00 \mathrm{Rf} 1.4 \mathrm{AU}$ & $2427.3 \mathrm{AU}$ & $5.13 \%$ & unknown ${ }^{2}$ \\
\hline
\end{tabular}

Track 1, ID: SFA5 Ethyl acetate extract

\begin{tabular}{|c|c|c|c|c|c|c|c|c|c|}
\hline Peak & $\begin{array}{c}\text { Start } \\
\text { Position }\end{array}$ & $\begin{array}{c}\text { Start } \\
\text { Height }\end{array}$ & $\begin{array}{c}\text { Max } \\
\text { Position }\end{array}$ & $\begin{array}{l}\text { Max } \\
\text { Height }\end{array}$ & $\begin{array}{l}\text { Max } \\
\%\end{array}$ & $\begin{array}{c}\text { End } \\
\text { End } \\
\text { Position } \\
\text { Height }\end{array}$ & Area & $\begin{array}{c}\text { Area } \\
\%\end{array}$ & Assigned substance \\
\hline 1 & $0.01 \mathrm{Rf}$ & $11.4 \mathrm{AU}$ & $0.02 \mathrm{Rf}$ & $85.7 \mathrm{AU}$ & $7.34 \%$ & $0.14 \mathrm{Rf} \quad 1.4 \mathrm{AU}$ & $2252.6 \mathrm{AU}$ & $7.74 \%$ & unknown ${ }^{*}$ \\
\hline 2 & $0.16 \mathrm{Rf}$ & $1.0 \mathrm{AU}$ & $0.23 \mathrm{Rf}$ & $236.4 \mathrm{AU}$ & $20.25 \%$ & $0.26 \mathrm{Rf} 94.8 \mathrm{AU}$ & $8030.2 \mathrm{AU}$ & $27.59 \%$ & unknown * \\
\hline 3 & $0.26 \mathrm{Rf}$ & $95.5 \mathrm{AU}$ & $0.30 \mathrm{Rf}$ & 644.9AU & $55.25 \%$ & $0.33 \mathrm{Rf} 34.1 \mathrm{AU}$ & 14605.0 AU & $50.17 \%$ & unknown * \\
\hline 4 & $0.33 \mathrm{Rf}$ & $34.1 \mathrm{AU}$ & $0.36 \mathrm{Rf}$ & 84.7AU & $7.25 \%$ & $0.37 \operatorname{Ri} 40.7 \mathrm{AU}$ & $1887.1 \mathrm{AU}$ & $6.48 \%$ & unknown * \\
\hline 5 & $0.37 \mathrm{Rf}$ & $42.2 \mathrm{AU}$ & $0.39 \mathrm{Rt}$ & $98.0 \mathrm{AU}$ & $8.40 \%$ & $0.45 \mathrm{Rf} \quad 0.0 \mathrm{AU}$ & $1886.2 \mathrm{AU}$ & $6.48 \%$ & unknown ${ }^{*}$ \\
\hline 6 & $0.94 \mathrm{Rf}$ & $0.1 \mathrm{AU}$ & $0.98 \mathrm{Rf}$ & $17.5 \mathrm{AU}$ & $1.50 \%$ & $1.00 \mathrm{Rf} 1.1 \mathrm{AU}$ & $448.0 \mathrm{AU}$ & $1.54 \%$ & unknown * \\
\hline
\end{tabular}

Figure 3: Rf values of ethyl acetate extract of SFA5 at UV $254 \mathrm{~nm}(\mathrm{~A})$ and $366 \mathrm{~nm}$ (B) 


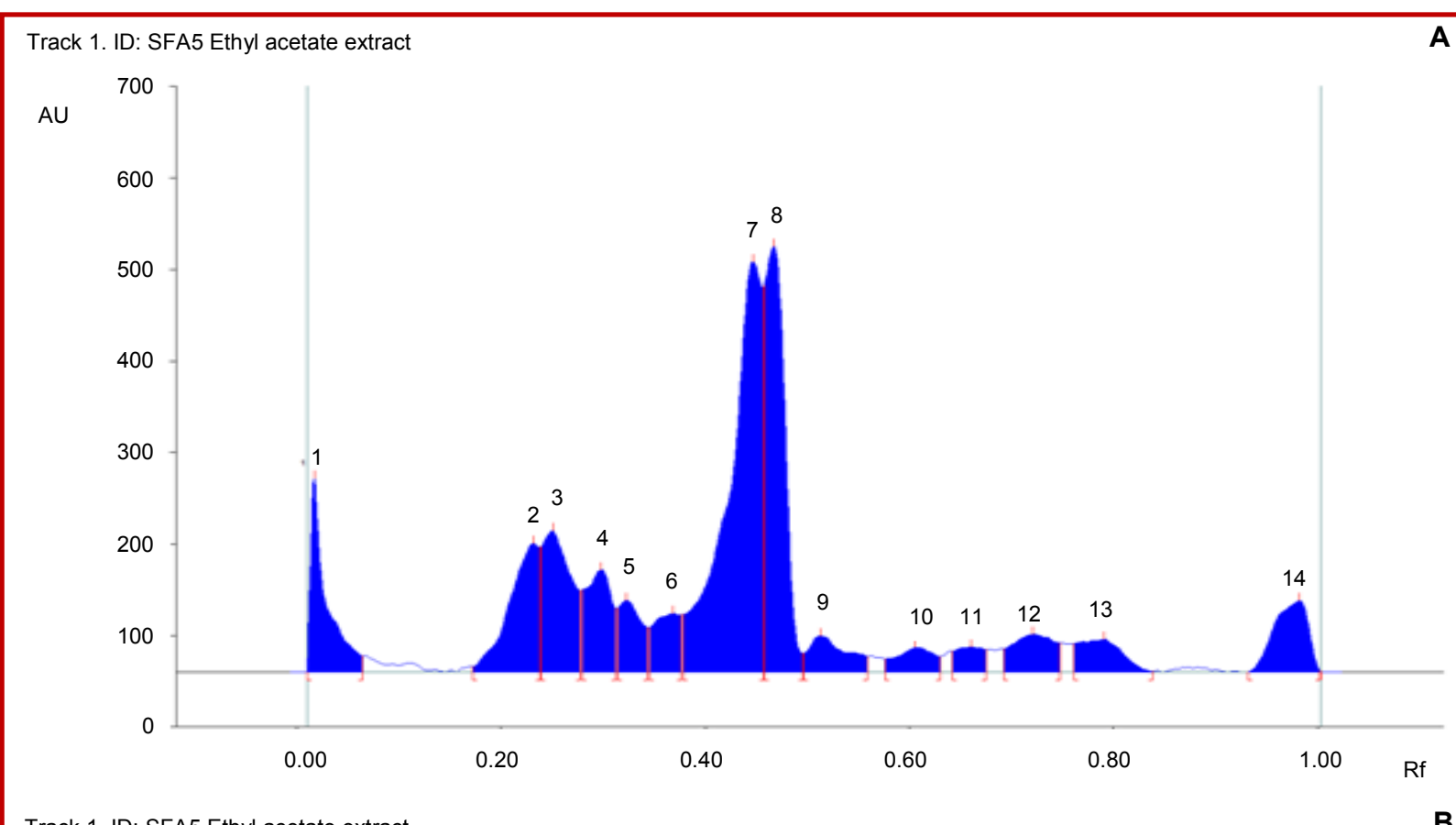

Track 1. ID: SFA5 Ethyl acetate extract

B

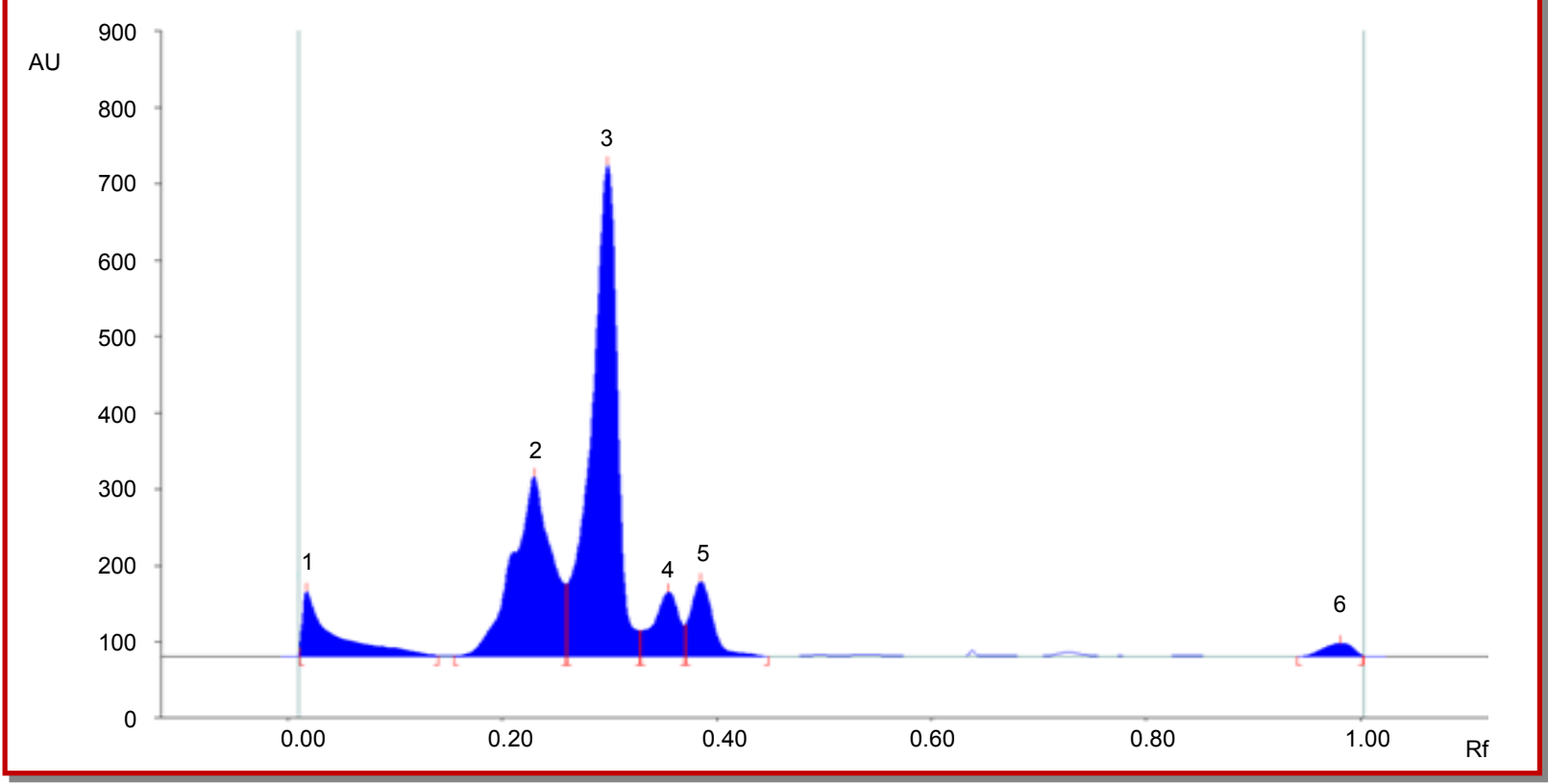

Figure 4: Finger print profile of ethyl acetate extracts of SFA5 at UV $254 \mathrm{~nm}$ (A) and $366 \mathrm{~nm}$ (B)

against Gram positive bacteria when compared to Gram negative bacteria (Berdy, 2005; Radhakrishnan et al., 2010). The antimicrobial activity of ethyl acetate extract confirmed the extracellular nature of bioactive compounds. Most of the bioactive metabolites from actionmycetes are extracellular in nature (Radhakrishnan et al., 2011). HPTLC is a reliable chromatographic method for analysing several samples of divergent nature and composition at the same time (Andola et al., 2010). HPTLC analysis of the ethyl acetate extract of SFA5 produced two yellow brown spots revealed the presence of two major antibacterial components at $R_{f}$ value of 0.38 and 0.46 in the chosen solvent system.

\section{Acknowledgement}

Authors thank the management of SSN College of Engineering for providing the research facilities.

\section{References}

Andola HC, Purohit VK. High performance thin layer chromatography (HPTLC): A modern analytical tool for 
biological analysis. Nature Sci. 2010; 8: 58-61.

Aravamuthan N, G Kumar, Karthik L, Bhaskara RKV. In vitro antagonistic activity of soil actinobacteria against multi-drug resistant bacteria. Pharmacologyonline 2010; 2: 507-16.

Baskar V, Madhanraj P, Kanimozhi K, Panneerselvam A. Characterization of carotenoids from selected strains of Streptomyces sp. Ann Biol Res. 2010; 1: 194-200.

Berdy J. Bioactive microbial metabolites. J Antibiot (Tokyo). 2005; 58: 1-26.

Chaudhary HS, Yadav J, Shrivastava AR, Singh S, Singh AK, Gopalan N. Antibacterial activity of actinomycetes isolated from different soil samples of Sheopur (a city of central India). J Adv Pharm Technol Res. 2013; 4: 118-23.

Febina BS, Kalidass S, Rachel RD. Qualitative analysis of antimicrobial compound by high performance thin layer chromatography method. Asian J Pharmaceut Clin Res. 2013; 6: 117-20.

Mamatha A. Quantitative HPTLC Analysis of Andrographolide in Andrographis paniculata obtained from different geographical sources (India). Int J Pharm Pharmaceut Sci. 2011; 3: 42-44.

Miyadoh S. Research on antibiotic screening in Japan over the last decade: A producing microorganisms approach. Actinomycetologica 1993; 9: 100-06.

Radhakrishnan M, Suganya S, Balagurunathan R, Vanaja K. Preliminary screening for antibacterial and antimycobacterial activity of actinomycetes from less explored ecosystems. World J Microbiol Biotechnol. 2010; 26: 561-66.

Radhakrishnan M, Balagurunathan R, Selvakumar N, Mukesh
D, Vanaja K. Bioprospecting of marine derived actinomycetes with special reference to antimycobacterial activity. Indian J Geo-Marine Sci. 2011; 40: 407-10.

Radhakrishnan M, Gopikrishnan V, Suresh A, Selvakumar N, Balagurunathan R, Vanaja K. Characterization and phylogenetic analysis of antituberculous compound producing actinomycete strain D25 isolated from Thar Desert Soil, Rajasthan. Bioinformation 2013; 9: 18-22.

Saravanan D, Bharathi S, Radhakrishnan M, Balagurunathan R. Exploitation of bacteria from forest ecosystem for antimicrobial compounds. J Appl Pharm Sci. 2012; 02: 120-23.

Shekar P, Anuradha J, Radhakrishnan M, Balagurunathan R. Effect of critical medium components on antimicrobial compound production from marine Streptomyces species (A2) by one factor at a time method. J Chem Pharm. 2011; 3: 87276 .

Shekar P, Sathish Kumar K, Anuradha J, Radhakrishnan M, Balagurunathan R. Optimization of medium components for antibacterial metabolite production from marine Streptomyces $S p$. PUA2 using response surface methodology. Int J Pharm Pharmaceut Sci. 2014; 6: 1-6.

Skoko N, Vujovic J, Savic M, Papic N, Vasiljevic B, Ljubijankic G. Construction of Saccharomyces cerevisiae strain FAV20 useful in detection of immunosuppressant's produced by soil actinomycetes, J Microbiol Methods. 2005; 61: 137-40.

Thakur D, Yadav A, Gogoi BK, Bora TC. Isolation and screening of Streptomyces in soil of protected forest areas from the states of Assam and Tripura, India, for antimicrobial metabolites. J de Mycologie Medicale. 2007; 17: 242 -49 . 


\section{Your feedback about this paper}

1. Number of times you have read this paper 0

2. Quality of paper Click

3. Your comments

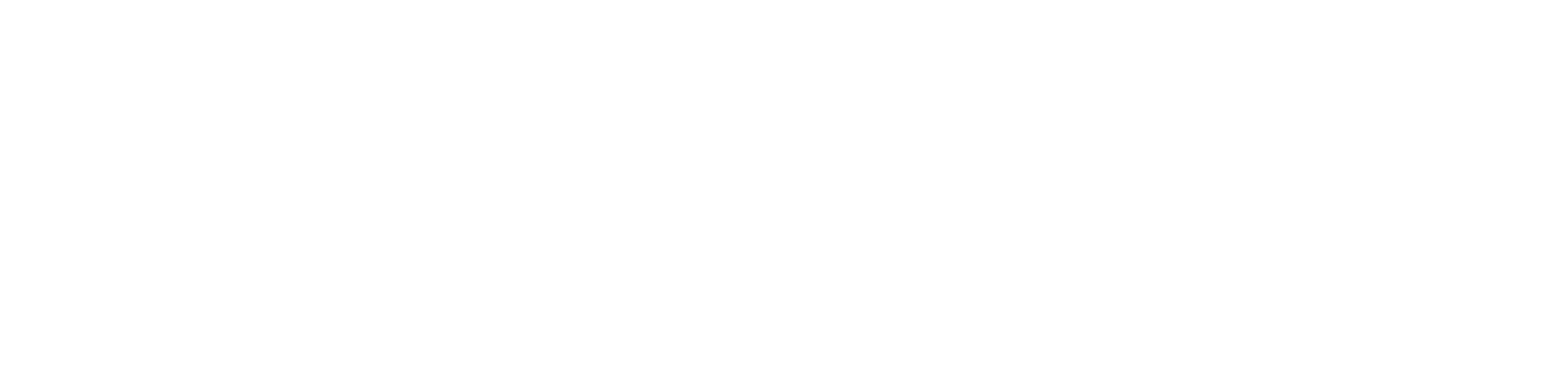

